\title{
Study on the effect of hydration on the shear strength of the interface between geosynthetic clay liner (GCL) and geomembrane
}

\author{
Xuan $\mathrm{Li}^{1, *}$, Yue Zhang ${ }^{2}$ \\ ${ }^{1}$ Hubei Business College, 430000 Wuhan, Hubei Province, China \\ ${ }^{2}$ Hubei Business College, 430000 Wuhan, Hubei Province, China
}

\begin{abstract}
Geosynthetic Clay Liner (GCL) and geomembrane is a kind of geosynthetics, which are widely used in large-scale landfills, road engineering and anti-seepage engineering, but the theoretical research of the two lags behind practice. The key to the overall stability of the landfill is the strength of the GCL shear strength. GCL has two types of shear failure: one is the interface shear strength failure, and the other is the GCL internal failure. In the existing failure cases of GCL used on slopes, it has not been found that the damage of GCL occurred inside the GCL. The key to maintaining the overall stability of the landfill is to determine the interface frictional shear strength of GCL, that is, the shear strength of the interface between GCL and other geotextiles at a certain slope position becomes a key factor in stability design. In this paper, under different hydration conditions of GCL, the influence of hydration solution, normal stress under hydration and hydration time on the shear strength of the interface between GCL and geomembrane is discussed.
\end{abstract}

\section{Introduction to the GCL}

Geosynthetic Clay Liner (GCL), a composite geosynthetic material, is developed on the basis of compacted clay liner (CCL) ${ }^{[1]}$. Compared with CCL, it has the characteristics of small unit area, high shear strength per unit thickness, low water permeability under the same conditions, strong self-healing ability, and strong ability to resist uneven settlement. Therefore, in a short period of time, the engineering field has been widely used in anti-seepage engineering and underground structure engineering ${ }^{[2]}$.

\section{GCL hydration principle}

Bentonite is a substance that swells rapidly when exposed to water, but when the type of bentonite is different, their swelling mechanism is also different. For calcium bentonite, the expansion mechanism is mainly manifested as internal crystal expansion; for sodium bentonite, the expansion mechanism is mainly manifested as osmotic expansion ${ }^{[3]}$.

The hydration of bentonite is a programmed process. The bentonite powder in the dry state exhibits a singlelayer structure, with extremely small thin layers and layers of water molecules separating the single-layer layers, and $\mathrm{Na}^{+}$appears on the surface of each layer. Positive ions are distributed in the medium, while negative ions are distributed on the surface of the thin layer, and cations absorb negative ions on the surface of the thin layer. After the cations in the medium contact water molecules, the water molecules will be adsorbed on the surface of the thin layer ${ }^{[4]}$. Since water molecules have a certain adsorption effect on water molecules, the water molecules adsorbed on the thin layer will adsorb more water molecules. The surface of the thin layer is negatively charged, and the adsorbed water molecules are connected and distributed on the surface of the thin layer ${ }^{[5]}$. Since the thin layers have the same charge, the newly formed thin layer will repel other thin layers, separating the two, causing expansion.

\section{The influence of hydration solution on the interfacial shear strength of GCL}

After GCL undergoes hydration in different solutions, the changes in bentonite are different. After the GCL sample is hydrated with ethanol, there is a lot of bentonite scattered in the solution; after the GCL sample is hydrated with deionized water, there is less bentonite scattered in the solution; after the GCL sample is hydrated with the $\mathrm{CaCl}_{2}$ solution, there is almost no scattered in the solution Bentonite. The thickness of the specimens after hydration of the three solutions did not change significantly. When the GCL sample is hydrated with deionized water, take the sample out and observe it. Experiment find-outs the bentonite particles between the intermediate layers condense into the gel layer. When the GCL sample is hydrated by the $\mathrm{CaCl}_{2}$ solution, the bentonite particles between the interlayers is still

\footnotetext{
* Corresponding author: lixuanfengguoer@126.com
} 
granular. And the granules are finer and uniform when they are dry. When the GCL sample is hydrated with ethanol, the bentonite particles between the interlayers have no obvious changes. As the shearing process continues, the hydration solution is squeezed out of the sample. The sample hydrated by the $\mathrm{CaCl}_{2}$ solution has a large amount of water; the degree of water output of deionized water is smaller than that of the sample hydrated by the $\mathrm{CaCl}_{2}$ solution; Due to the strong volatility of ethanol, during the test preparation process, its water content decreases rapidly, and the sample has the smallest water output.

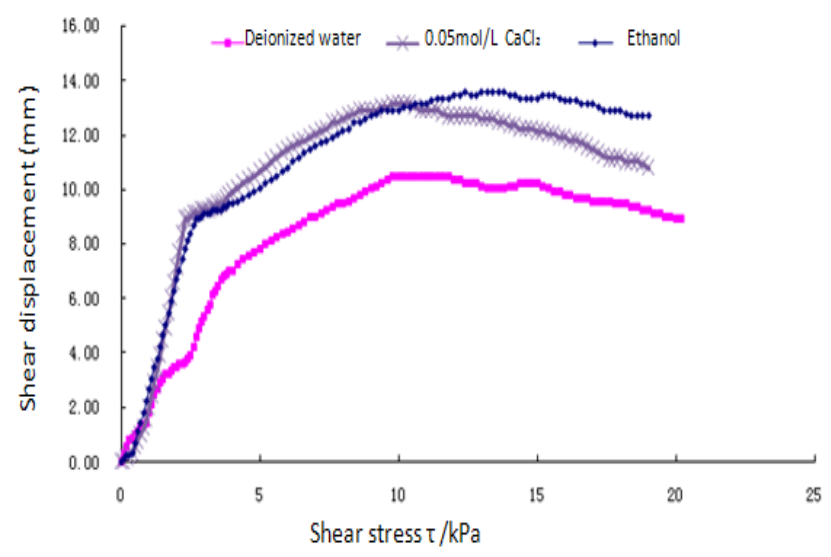

Fig. 1. The relationship graph of the influence of different hydration solutions (Deionized water, $0.05 \mathrm{~mol} / \mathrm{L} \mathrm{CaCl}_{2}$ solution, ethanol) on the shear strength of GCL

It can be seen from Fig. 1 that the shear stress of GCL after hydration with deionized water and $0.05 \mathrm{~mol} / \mathrm{L}$ $\mathrm{CaCl}_{2}$ solution increases. That is, as the ion concentration increases, the shear stress increases accordingly. The reason is that the higher the ion concentration, the lower the water content of the sample after hydration, and the greater the consistency of the gel after hydration of bentonite, which has a greater shear force. The shear stress of the GCL sample hydrated by ethanol is greater than that of the GCL sample hydrated by deionized water. But compared with the GCL sample hydrated by the $0.05 \mathrm{~mol} / \mathrm{L} \mathrm{CaCl}_{2}$ solution, the peak intensity does not show obvious regularity, can not be compared directly.

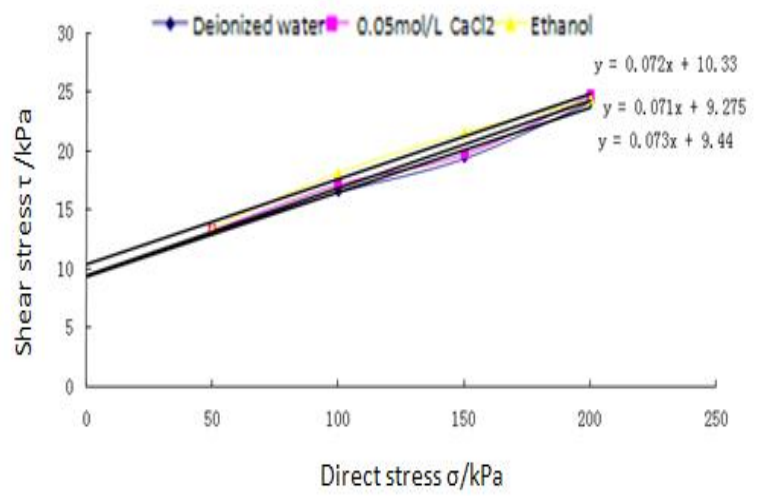

Fig. 2. GCL and geomembrane maximum shear stress and normal stress $\left(\tau_{f}-p\right)$ relationship graph
Table 1. Test results of interface friction between different hydration solutions GCL and geomembrane

\begin{tabular}{|c|c|c|c|}
\hline \multirow{2}{*}{$\begin{array}{c}\text { Geotechnical } \\
\text { materials }\end{array}$} & $\begin{array}{c}\text { Hydration } \\
\text { solution }\end{array}$ & Cohesion & $\begin{array}{c}\text { Friction } \\
\text { angle }\end{array}$ \\
\cline { 3 - 4 } & $\mathrm{c} / \mathrm{kPa}$ & $\varphi\left({ }^{\circ}\right)$ \\
\hline \multirow{2}{*}{$\begin{array}{c}\text { GCL and } \\
\text { geomembrane }\end{array}$} & $\begin{array}{c}0.05 \mathrm{~mol} / \mathrm{L} \\
\mathrm{CaCl} 2 \\
\text { solution }\end{array}$ & 9.44 & $3.43^{\circ}$ \\
\cline { 2 - 4 } & ethanol & 10.33 & $4.11^{\circ}$ \\
\hline
\end{tabular}

The direct shear test results of the GCL and geomembrane interface friction after hydration with different hydration solutions are shown in Figure 2 and Table 1. Comprehensive research and analysis can draw the following conclusion: GCL sample interface shear strength is different after different solution hydration. Under pressureless hydration conditions, the cohesive force of interfacial friction between GCL and geomembrane is represented by ethanol solution $>0.05 \mathrm{~mol} / \mathrm{L} \mathrm{CaCl}_{2}$ solution $>$ deionized water, and the internal friction angle is represented by deionized water $>0.05 \mathrm{~mol} / \mathrm{L} \quad \mathrm{CaCl}_{2}$ solution $>$ Ethanol, under pressureless hydration conditions, the shear resistance of the interface friction between GCL and geomembrane is ethanol $>0.05 \mathrm{~mol} / \mathrm{L} \mathrm{CaCl} 2$ solution $>$ deionized water.

\section{The influence of hydration normal stress state on the interfacial shear strength of GCL}

The experiment selected three different hydration normal stress states as shown in Figure 3 (the hydration solution is deionized water).

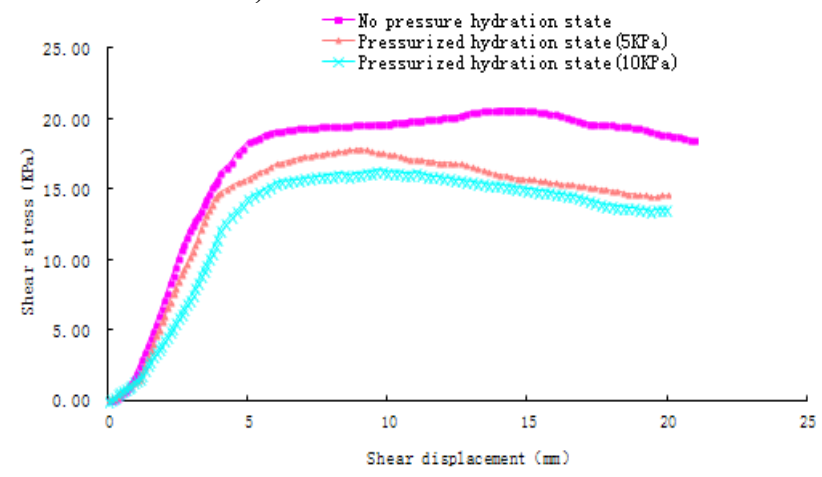

Fig. 3. Different hydration normal stress conditions of (pressureless hydration condition, $5 \mathrm{kPa}$ hydration condition and $10 \mathrm{kPa}$ hydration condition) GCL and geomembrane graph

It can be seen from Fig. 3 that under the same normal stress, the GCL peak intensity pressureless hydration state $>5 \mathrm{kPa}$ normal stress hydration state $>10 \mathrm{kPa}$ normal stress hydration state. It can be seen that the shear stress of the interface between the GCL and the 
geomembrane after hydration with deionized water increases with the increase of the hydration normal stress, and the shear stress decreases.

Table 2. The peak value of shear stress at the interface friction between GCL and geomembrane under different hydration conditions

\begin{tabular}{|c|c|c|c|c|}
\hline Normal stress & $50 \mathrm{kPa}$ & $100 \mathrm{kPa}$ & $150 \mathrm{kPa}$ & $200 \mathrm{kPa}$ \\
\hline $\begin{array}{c}\text { Pressureless } \\
\text { hydration }\end{array}$ & 13.11 & 16.44 & 19.33 & 24.11 \\
\hline $\begin{array}{c}\mathbf{5 k P a} \\
\text { Hydration } \\
\text { conditions }\end{array}$ & 10.44 & 13.11 & 16.33 & 19.22 \\
\hline $\begin{array}{c}\mathbf{1 0 k P a} \\
\text { Hydration } \\
\text { conditions }\end{array}$ & 11.78 & 14.33 & 17.89 & 20.67 \\
\hline
\end{tabular}

Analyzed by Figure 4 and Table 3: GCL and geomembrane interface friction cohesion pressureless hydration condition $>5 \mathrm{kPa}$ normal stress hydration condition $>10 \mathrm{kPa}$ normal stress hydration condition; and under the three conditions, GCL and the rough surface film interface friction The number of internal friction angles of pressureless hydration conditions $>5 \mathrm{kPa}$ normal stress hydration conditions $>10 \mathrm{kPa}$ normal stress hydration conditions. In comparison, the shear strength of the interface between GCL and geomembrane is pressureless hydration condition $>5 \mathrm{kPa}$ normal stress hydration condition $>10 \mathrm{kPa}$ normal stress hydration condition.

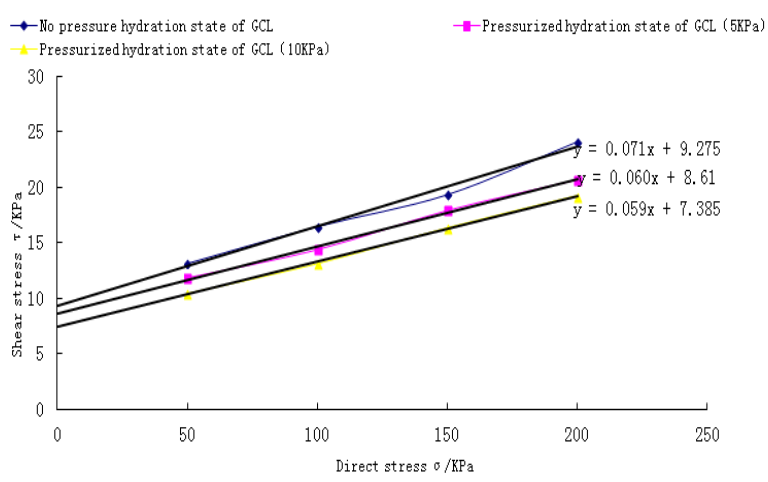

Fig. 4. GCL and geomembrane maximum shear stress and normal stress $\left(\tau_{f}-p\right)$ relationship graph

Table 3. Test results of interface friction between different hydration solutions GCL and geomembrane

\begin{tabular}{|c|c|c|c|}
\hline \multirow{2}{*}{$\begin{array}{c}\text { Geotechnical } \\
\text { materials }\end{array}$} & $\begin{array}{c}\text { Hydration } \\
\text { solution }\end{array}$ & Cohesion & $\begin{array}{c}\text { Friction } \\
\text { angle }\end{array}$ \\
\cline { 3 - 4 } & $\mathrm{c} / \mathrm{kPa}$ & $\varphi\left({ }^{\circ}\right)$ \\
\hline \multirow{2}{*}{$\begin{array}{c}\text { Geionized } \\
\text { water }\end{array}$} & 9.275 & $4.11^{\circ}$ \\
\cline { 2 - 4 } geomembrane & $\begin{array}{c}0.05 \mathrm{~mol} / \mathrm{L} \\
\mathrm{CaCl} 2 \\
\text { solution }\end{array}$ & 8.61 & $3.43^{\circ}$ \\
\cline { 2 - 4 } & ethanol & 7.385 & $3.38^{\circ}$ \\
\hline
\end{tabular}

From this, it can be concluded that the influence of different hydration stress on the shear strength is: under the same state, the friction strength between the GCL and the geomembrane decreases with the increase of the hydration normal stress.

\section{The influence of hydration time on the interfacial shear strength of GCL}

During the hydration process, the GCL samples after different hydration times were observed and the water content was measured (Figure 5 and Table 5): The water content of the GCL sample after hydration with deionized water for day 1 It was $168.3 \%, 181.9 \%$ after 5 days, and $194.7 \%$ after 10 days.

Table 4. GCL water content after different hydration time

\begin{tabular}{|c|c|c|c|c|}
\hline $\begin{array}{c}\text { Hydration } \\
\text { solution }\end{array}$ & $0 \mathrm{~d}$ & $1 \mathrm{~d}$ & $5 \mathrm{~d}$ & $10 \mathrm{~d}$ \\
\hline $\begin{array}{c}\text { Deionized } \\
\text { water }\end{array}$ & $16.2 \%$ & $168.3 \%$ & $181.9 \%$ & $194.7 \%$ \\
\hline
\end{tabular}

The degree of hydration of bentonite causes the effect of hydration time on the shear strength of GCL. From the previous analysis, it can be seen that the bentonite in the dry state is granular, while the bentonite particles after hydration are gelatinous. And during the hydration process, the bentonite swells, and its volume can increase up to 24 times. Because the bentonite of the GCL sample is fixed on the interlayer of the geotextile by needle punching, the bentonite that is transformed into a gel is bound to be squeezed and tighter, thereby preventing water penetration. The longer the hydration time, the more water molecules will enter the bentonite, which will dilute the gel to a certain extent, and the friction will decrease as the consistency of the gel decreases. The water content of the corresponding GCL under different hydration times proves this point: the water content of the GCL after 1 day of free hydration is $168.3 \%$, and the water content of the GCL after 10 days of free hydration increases to $194.7 \%$.

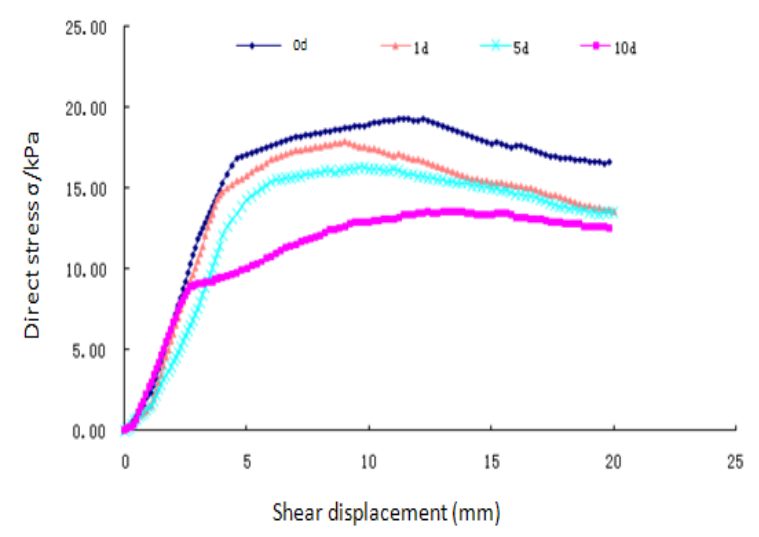

Fig. 5. The relationship graph of the influence of different hydration time $(0 \mathrm{~d}, 1 \mathrm{~d}, 5 \mathrm{~d}, 10 \mathrm{~d})$ on the shear strength of GCL

In the dry state, the cohesive force and internal friction angle of the interface friction between GCL and 
the rough mask are the largest (shown in Table 5 and Figure 6), and the cohesive force and internal friction angle of the interface friction between GCL and the rough mask after a day of hydration are the second, GCL after ten days of hydration has the smallest cohesive force and internal friction angle of friction between the GCL and the rough mask.

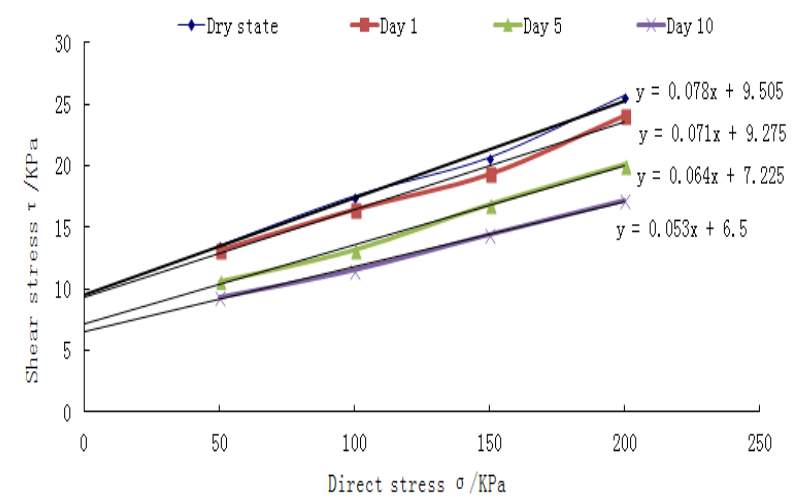

Fig. 6. GCL and geomembrane maximum shear stress and normal stress $\left(\tau_{f}-p\right)$ relationship graph

Table 5. Test results of interface friction between different hydration solutions GCL and geomembrane

\begin{tabular}{|c|c|c|c|}
\hline \multirow{4}{*}{$\begin{array}{c}\text { Geotechnical } \\
\text { materials }\end{array}$} & $\begin{array}{c}\text { Hydration } \\
\text { solution }\end{array}$ & Cohesion & $\begin{array}{c}\text { Friction } \\
\text { angle }\end{array}$ \\
\cline { 3 - 4 } & $\mathrm{c} / \mathrm{kPa}$ & $\varphi\left({ }^{\circ}\right)$ \\
\hline \multirow{4}{*}{$\begin{array}{c}\text { GCL and } \\
\text { geomembrane }\end{array}$} & Day 0 & 9.505 & $4.46^{\circ}$ \\
\cline { 2 - 4 } & Day 1 & 9.275 & $4.11^{\circ}$ \\
\cline { 2 - 4 } & Day 10 & 7.225 & $3.66^{\circ}$ \\
\cline { 2 - 4 } & 6.500 & $3.03^{\circ}$ \\
\hline
\end{tabular}

It can be concluded that the influence of hydration time on shear strength is: The shear strength of GCL decreases with the increase of hydration time; the effect of hydration time gradually becomes smaller as time continues.

\section{Summary}

The conclusions obtained through the above-mentioned experimental analysis are as follows:

1.The shear strength of GCL samples after hydration with different solutions is different. The shear strength of GCL samples hydrated by $0.05 \mathrm{~mol} / \mathrm{L} \mathrm{CaCl}_{2}$ solution and ethanol is stronger than that of GCL samples hydrated by deionized water;

2.Under the same condition, the frictional shear strength of the interface between GCL and geomembrane decreases with the increase of hydration normal stress;

3. The shear strength of GCL decreases with the increase of hydration time; the influence of hydration time gradually becomes smaller as time continues.

\section{References}

1. X. Li, Laboratory investigation on the interface of GCL and Geomembrane. (2013)

2. Y. Duan, L. Hou, Z. Shi, Z. Zhang, N. Li. China Mining. 04, 255-257+262(2020)

3. J. He, X. Wang, X. Yan, J. Wan, Z. Zhu. Journal of Jilin University. 03, 807-816(2019)

4. X. Kong, Experimental study on the anti-seepage performance of GCL in liner of waste landfill. (2011)

5. J. Zhou, Environmental Geotechnical Engineering. (2009) 